\title{
Hyperactivity in the Ventral Cochlear Nucleus after Cochlear Trauma
}

\author{
Darryl P. Vogler, Donald Robertson, and Wilhelmina H. A. M. Mulders \\ The Auditory Laboratory, School of Biomedical, Biomolecular, and Chemical Sciences, The University of Western Australia, Crawley, Western Australia \\ 6009 , Australia
}

The emergence of hyperactivity in the form of elevated spontaneous firing rates after cochlear trauma has been well documented in a number of central auditory structures, including the auditory cortex, inferior colliculus, and dorsal subdivision of the cochlear nucleus. This hyperactivity is of interest as a possible neural substrate of tinnitus. Whether the ventral subdivision of the cochlear nucleus shows hyperactivity has never been investigated despite the fact that, like the dorsal division, it also receives direct input from the damaged cochlea and supplies major ascending inputs to brainstem and midbrain auditory centers. We investigated spontaneous neuronal firing rates in the ventral cochlear nucleus in a guinea pig model of cochlear trauma in which we have shown that hyperactivity consistently develops in the inferior colliculus (Mulders and Robertson, 2009). The mean spontaneous firing rates of ventral cochlear nucleus neurons was significantly elevated compared to sham controls. This hyperactivity was more evident in primary-like and onset categories of neurons. Hyperactivity in the ventral subdivision of cochlear nucleus therefore needs to be considered in relation to neural models of the genesis of tinnitus.

\section{Introduction}

The presence of elevated levels of spontaneous neural firing (hyperactivity) after treatments that are associated with the development of tinnitus has been well documented in several stages of the central auditory pathways, including the auditory cortex, inferior colliculus, and cochlear nucleus (Kaltenbach and Afman, 2000; Komiya and Eggermont, 2000; Brozoski et al., 2002; Seki and Eggermont, 2003; Kaltenbach et al., 2004; Ma et al., 2006; Bauer et al., 2008; Mulders and Robertson, 2009; Dong et al., 2010). The cochlear nucleus $(\mathrm{CN})$ is the first brainstem auditory nucleus, and elevated activity at this level is of interest because it could either directly drive the hyperactivity seen in higher centers or, alternatively, it could trigger mechanisms that eventually lead to the de novo generation of hyperactivity in the higher parts of the pathway.

Changes in neuronal electrical activity in the dorsal subdivision of the cochlear nucleus (DCN) after treatments known to induce tinnitus have been thoroughly documented (Kaltenbach and Afman, 2000; Brozoski et al., 2002; Kaltenbach et al., 2004), but the ventral subdivision (VCN) has received little attention. This is surprising in view of the fact that, like the DCN, the VCN receives input from all primary afferent auditory nerve fibers and

\footnotetext{
Received Dec. 14, 2010; revised Feb. 14, 2011; accepted March 12, 2011

Author contributions: D.R. and W.H.A.M.M. designed research; D.P.V. performed research; D.P.V. analyzed data; D.R. and W.H.A.M.M. wrote the paper.

This work was supported by grants from the Royal National Institute for Deaf People (UK), the Medical and Health Research Infrastructure Fund, and The University of Western Australia. We thank M. Lloyd and I. M. Winter for generous provision of data acquisition software and I. M. Winter for the MATLAB routines.

Correspondence should be addressed to Wilhelmina H. A. M. Mulders, The Auditory Laboratory, Discipline of Physiology, School of Biomedical, Biomolecular and Chemical Sciences M311, The University of Western Australia, 35 Stirling Highway, Crawley Western Australia 6009, Australia. E-mail: hmulders@cyllene.uwa.edu.au.

DOI:10.1523/JNEUROSCI.6538-10.2011

Copyright $\odot 2011$ the authors $\quad 0270-6474 / 11 / 316639-07 \$ 15.00 / 0$
}

provides major direct and indirect pathways to higher auditory centers. Salvi et al. (1978) reported a decrease in spontaneous firing rates in VCN, but this study only measured activity a short time after a relatively mild sound exposure and measured mainly from low-frequency neurons (64\% lower than $4 \mathrm{kHz}$ ). Imig and Durham (2005) reported alterations in the regional distribution of deoxyglucose uptake in cat VCN 1 week after unilateral noise exposure, but changes in absolute levels of uptake were not evident. Another indirect measure of neural activity, manganeseenhanced magnetic resonance imaging, was reported by Brozoski et al. (2007), who showed increases in posteroventral cochlear nucleus in rats with behaviorally demonstrated tinnitus. Zheng et al. (2006) reported an increase in the number of nitric oxide synthase-expressing neurons in VCN, but not DCN, after treatment with the tinnitus-inducing drug salicylate, suggesting an upregulation of excitatory processes specific to VCN.

However, none of these previous studies have directly investigated neuronal electrical activity in the VCN after tinnitusinducing treatments. In the present study therefore, we measured single neuron spontaneous firing rates in a large number of neurons in guinea pig VCN after restricted cochlear trauma that we have previously shown to induce hyperactivity in the auditory midbrain (Dong et al., 2009, 2010; Mulders and Robertson, 2009; Mulders et al., 2010). As in our previous studies in the inferior colliculus, we investigated whether the mechanism of cochlear trauma, mechanically and acoustically induced, affects the level and pattern of any hyperactivity observed. Finally, we investigated whether different cell types in VCN were differentially affected by acoustic trauma. It is well known that the different cell types form multiple parallel outputs from the VCN with different roles in auditory signal processing (for review, see Cant and Benson, 2003). We found a significant elevation of spontaneous fir- 
ing rates in VCN after cochlear trauma, and this increase may be more evident in particular neuronal response categories in VCN.

\section{Materials and Methods}

Animals. Results are reported from 19 adult pigmented guinea pigs of either sex weighing between 260 and $315 \mathrm{~g}$ at the time of initial surgery. The experimental protocols conformed to the Code of Practice of the National Health and Medical Research Council of Australia and were approved by the Animal Ethics Committee of The University of Western Australia.

Initial surgery for cochlear trauma and sham controls. Following a subcutaneous injection of $0.1 \mathrm{ml}$ of atropine sulfate $(0.6 \mathrm{mg} / \mathrm{ml})$, animals received an intraperitoneal injection of diazepam $(5 \mathrm{mg} / \mathrm{kg})$, followed 20 min later by an intramuscular injection of Hypnorm $(0.315 \mathrm{mg} / \mathrm{ml}$ fentanyl citrate and $10 \mathrm{mg} / \mathrm{ml}$ fluanisone; $1 \mathrm{ml} / \mathrm{kg}$ ). When deep anesthesia was obtained as determined by the absence of the foot withdrawal reflex, animals were placed on a heating blanket in a soundproof room and mounted in hollow ear bars. A small opening was made in the bulla to place an insulated silver wire on the round window. A compound action potential (CAP) audiogram (Johnstone et al., 1979) for the frequency range $4-24 \mathrm{kHz}$ was recorded to assess the animals' cochlear sensitivity using methods described in detail previously (Mulders and Robertson, 2009). All sound stimuli were presented using a closed sound system through a 0.5 inch condenser microphone driven in reverse as a speaker (Bruel and Kjaer, type 4134). Pure tone stimuli were synthesized by a computer equipped with DIGI96 soundcard connected to an analog/ digital interface (ADI-9 DS, RME Intelligent Audio Solution). Sample rate was $96 \mathrm{kHz}$. The interface was driven by a custom-made computer program (Neurosound, MI Lloyd), which was also used to collect single neuron data. CAP signals were amplified, filtered $(100 \mathrm{~Hz}-3 \mathrm{kHz}$ bandpass), and recorded with a second data acquisition system (Powerlab $4 \mathrm{SP}, \mathrm{AD}$ Instruments). If cochlear sensitivity was within the normal range, the animal was randomly assigned to one of three groups. Group 1 received no further treatment and served as sham controls $(n=6)$. In group $2(n=6)$, the contralateral ear was blocked with plasticine and the animal was subjected to an acoustic trauma (a continuous loud tone for $2 \mathrm{~h}, 10 \mathrm{kHz}, 124 \mathrm{~dB}$ sound pressure level). In group $3(n=7)$, a mechanical lesion was made in the basal turn of the cochlea using the methods described in detail by Robertson et al. (1980). After the acoustic trauma or mechanical trauma, another CAP audiogram was measured to assess the immediate impact on peripheral sensitivity. In all groups, the wound was sutured and buprenorphine $(0.05 \mathrm{mg} / \mathrm{kg}$ s.c.) was given postoperatively as analgesic. Animals were allowed to recover and were kept for 2 weeks before the final single neuron recordings.

Surgery for final experiments. Anesthesia consisted of a subcutaneous injection with $0.1 \mathrm{ml}$ atropine followed by an intraperitoneal injection of Nembutal (pentobarbital sodium, $30 \mathrm{mg} / \mathrm{kg}$ ) and a $0.15 \mathrm{ml}$ intramuscular injection of Hypnorm. Maintenance anesthesia regime consisted of full Hypnorm doses every hour and half doses of Nembutal every $2 \mathrm{~h}$. Animals were placed on a heating blanket in a sound proof room and artificially ventilated on carbogen $\left(95 \% \mathrm{O}_{2}\right.$ and $\left.5 \% \mathrm{CO}_{2}\right)$. Paralysis was induced with $0.1 \mathrm{ml}$ pancuronium bromide $(2 \mathrm{mg} / \mathrm{ml}$ i.m. $)$. The electrocardiogram was continuously monitored and heart rate never increased over pre-paralysis levels at any stage of the experiments. After the animals were mounted in hollow ear bars, the left cochlea was exposed and the CAP audiogram was recorded with a silver wire placed on the round window as for the initial experiment. All sound stimuli during single neuron recordings were delivered using methods identical to those employed in the initial CAP measurements.

To obtain extracellular single neuron recordings from the $\mathrm{CN}$, a posterior craniotomy was performed and the caudal and lateral aspects of the left cerebellum were aspirated to visualize the DCN. A glass-insulated tungsten microelectrode (Merrill and Ainsworth, 1972) was advanced using a stepping motor microdrive at an angle of $\sim 45^{\circ}$ to the horizontal plane through the more rostral portion of the DCN while monitoring cluster and single neuron responses to a broad band noise burst. Electrode placement in the VCN was signaled by a sudden transition from low to high-frequency characteristic frequency $(\mathrm{CF})$ and a large robust
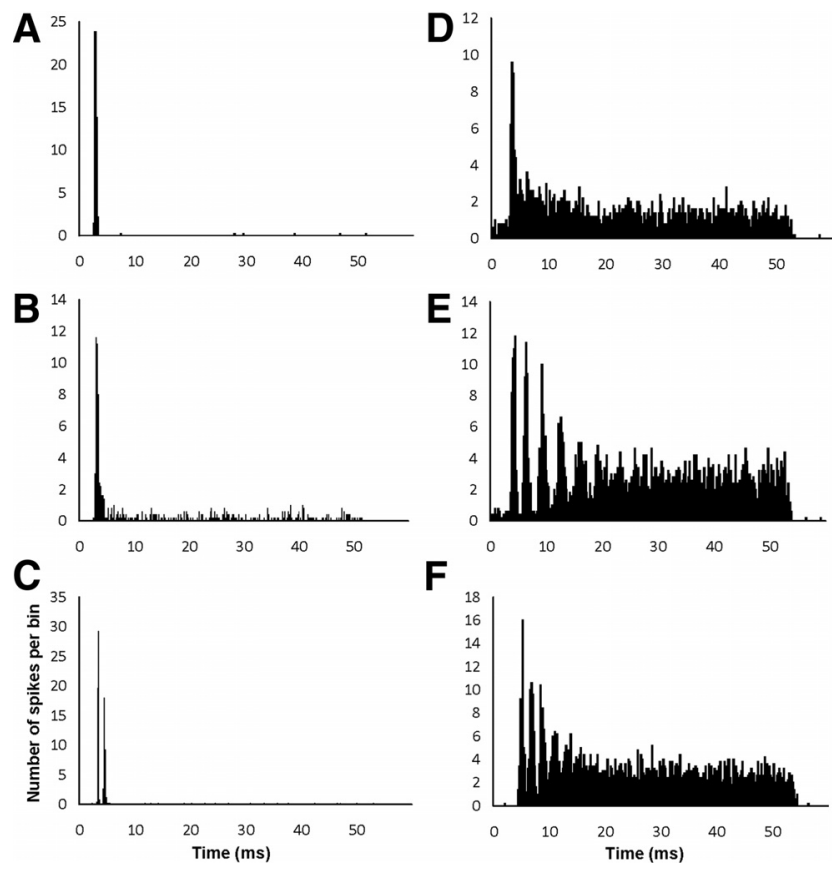

Figure 1. Examples of peristimulus-time histograms from sham animals in ventral cochlear nucleus. Bin size $0.2 \mathrm{~ms}$. $\boldsymbol{A}-\boldsymbol{F}$, Different response types based on PSTH shape. $A, 0 \mathrm{i}, \mathrm{CF}=10 \mathrm{kHz}$. $B, 0 \mathrm{~L}, \mathrm{CF}=19.9 \mathrm{kHz} . \mathrm{C}, 0 \mathrm{C}, \mathrm{CF}=2.5 \mathrm{kHz} ; \boldsymbol{D}, \mathrm{PL}, \mathrm{CF}=2.7 \mathrm{kHz} ; \boldsymbol{E}, \mathrm{Cs}, \mathrm{CF}=1.7 \mathrm{kHz} . \boldsymbol{F}, \mathrm{Ct}, \mathrm{CF}=$ $0.7 \mathrm{kHz}$.

short latency field potential. The cavity overlying the $\mathrm{CN}$ was then filled with $4 \%$ agar in saline to ensure mechanical stability.

Single neuron recordings and classification. In each electrode track through the VCN a broadband noise stimulus (50 ms duration) was employed throughout as a search stimulus. This was done to ensure that all response types would generate driven activity, so as to reduce the possibility of sampling bias in the recordings. Furthermore, data were obtained from all neurons encountered that showed spikes that were clearly distinguishable from the background electrical activity. Each electrode track through the VCN was continued until a reduction in field potential amplitude and cluster responses indicated that the electrode was exiting the VCN. The electrode was then withdrawn and repositioned and a new track was begun. This procedure was continued until CAP threshold measurements indicated a deterioration of cochlear sensitivity.

When a single neuron was isolated, its CF and threshold at CF were determined audio-visually. The spontaneous firing rate (SFR) was then measured for a period of $10 \mathrm{~s}$. During this measurement the input to the speaker was turned off to reduce any background "hiss" that may have been emanating from the sound system.

Neurons were classified according to a number of criteria developed by others (Rhode et al., 1983; Rhode and Smith, 1986; Young et al., 1988; Winter and Palmer, 1990, 1995). Examples of peristimulus time histograms (PSTHs) for the major response categories are shown in Figure 1. PSTHs were constructed from 250 stimulus presentations at CF $(50 \mathrm{~ms}$ duration; repetition rate, 4/s) $20 \mathrm{~dB}$ above CF threshold. Input-output curves were constructed to either tone bursts at CF (50 ms duration) or broadband noise (50 ms duration; bandwidth, $0-20 \mathrm{kHz}$ ) in $5 \mathrm{~dB}$ increments of random order from below threshold to the highest intensity where spikes were still distinguishable

Neurons were classified into five categories: primary-like (PL), primary-like with notch (PLn), onset (O), sustained chopper (Cs), and transient chopper $(\mathrm{Ct})$. An offline analysis of all PSTH raw data was conducted using MATLAB (version 6.5). Each PSTH was plotted over a $60 \mathrm{~ms}$ temporal time span, which included the $50 \mathrm{~ms}$ delivery of the tone and $10 \mathrm{~ms}$ of the off response after the termination of the tone. The spikes were grouped in $0.2 \mathrm{~ms}$ blocks over the $60 \mathrm{~ms}$. Neurons were classified as onset neurons when the onset peak was $>10 \times$ the steady-state firing 

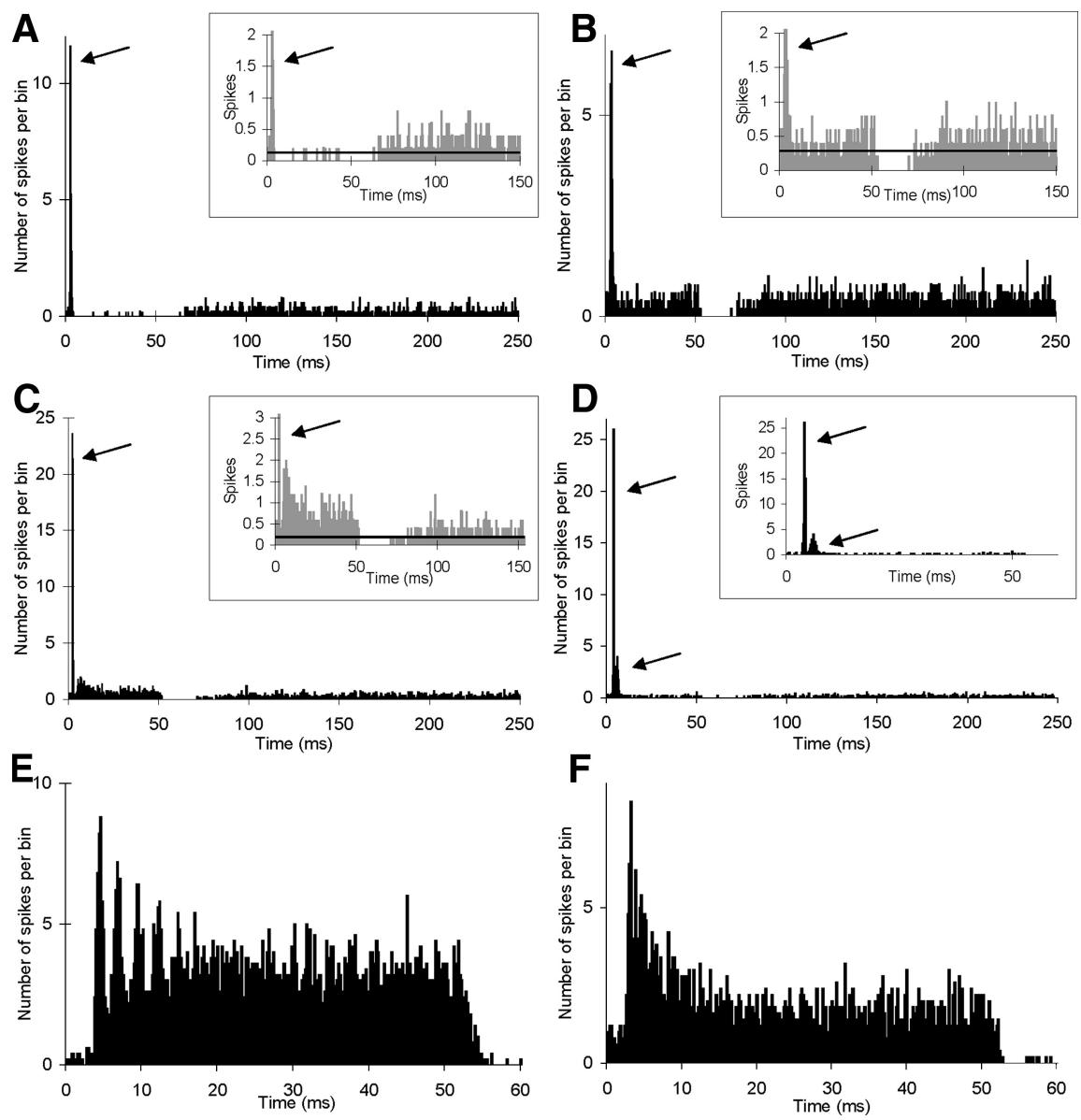

Figure 2. Examples of different response categories of neurons found in animals subjected to mechanical or acoustic trauma. $\boldsymbol{A}-\boldsymbol{D}$, Examples of PSTHs of onset neurons, all with abnormally high spontaneous firing rates. Arrows indicate onset peaks. Inserts show same PSTH with expanded $x$ - and $y$-axes. Horizontal lines in inserts show average spontaneous firing rate, estimated from $150-250$ ms time bins. $\boldsymbol{A}, \boldsymbol{B}$, Two examples of neurons classified as $0 \mathrm{i}$. $\boldsymbol{C}$, Neuron classified as $0 \mathrm{~L}$. $\boldsymbol{D}$, Neuron classified as 0 c. $\boldsymbol{E}, \boldsymbol{F}$ Examples of neurons classified as sustained chopper and primary-like, respectively. Unit $C F: A, 9.3 \mathrm{kHz} ; \boldsymbol{B}, 13 \mathrm{kHz} ; \boldsymbol{C}, 19.7 \mathrm{kHz} ; \boldsymbol{D}, 1.2$ $\mathrm{kHz} ; \boldsymbol{E}, 4.8 \mathrm{kHz} ; \boldsymbol{F}, 2.2 \mathrm{kHz}$.

level. Where possible, onset neurons were further classified as Oi, which showed no steady-state firing after the onset peak (Fig. $1 \mathrm{~A}$ ), OL, which showed some steady-state firing after the onset peak (Fig. $1 B$ ), or onset chopper neurons $(\mathrm{Oc})$, which showed two peaks and responded better to noise than CF tones (Fig. 1C) (Winter and Palmer, 1995; Arnott et al., 2004). Chopper and primary-like neurons were distinguished from each other on the basis of coefficient of variation (regularity index) (Young et al., 1988). Cs show a coefficient of variation $(\mathrm{CV})<0.25$ (Fig. $1 E$ ), Ct show a CV between 0.25 and 0.5 (Fig. $1 F$ ), and PL show a CV $>0.5$ (Fig. $1 D$ ).

Figure 2 shows examples of the different response categories in animals subjected to prior cochlear trauma. In the case of Oi and OL categories (Fig. $2 \mathrm{~A}-\mathrm{C}$ ), the PSTH classification required some adjustment in animals because of the observed increases in spontaneous firing rate. Both of these onset categories normally show very low or zero spontaneous firing rate. When spontaneous firing rate was elevated in onset neurons, the neurons were classified after comparing firing during the tone to the spontaneous rate (Fig. $2 A-C$, see insets). Neurons showing significant sound-evoked firing above the spontaneous firing rate during the main part of the tone were classified as OL (Fig. $2 C$ ), while those that did not were classified as $\mathrm{Oi}$ (Fig. $2 A, B$ ). Oc neurons still showed at least two distinct early peaks in their PSTHs (Fig. $2 D$ ), very wide dynamic range and higher firing rates to noise than to pure tone stimuli (Winter and Palmer, 1995; Arnott et al., 2004; Mulders et al., 2007). Chopper-type and primary-like categories were easily distinguishable in these animals regardless of spontaneous firing rate (Fig. 2E,F).
For a number of neurons, although SFR could be obtained, a number of factors prevented complete data collection. This was the case when a neuron was lost before CF or PSTH was obtained or when the spike height was not sufficiently large to be distinguishable from the field potentials that were particularly present at high stimulus intensities. These neurons remained unclassified but their SFR was included in the gross comparison between groups, but not in further analysis relating to CF and response category. Another group of neurons, even though the PSTH was obtained, could not be unequivocally assigned to any response category and were deemed unclassifiable. The proportion of these latter neurons was low (see Table 1) and did not differ significantly between the three groups.

Statistics. For simple statistical analysis between two normally distributed groups, as in the comparison of CAP amplitudes, $t$ tests and one-way ANOVAs from Microsoft Excel and Graph Pad Prism were used. Due to the nonGaussian distribution of the spontaneous rates, analysis was completed in GraphPad prism (version 5.0) using Kruskal-Wallis one-way ANOVA for multiple groups or a Mann-Whitney test for a two-group comparison. $\chi^{2}$ tests were also conducted for the comparison between the percentages of cell types and the comparison of spontaneous firing rate distribution in each condition.

\section{Results}

\section{Cochlear thresholds}

Figure 3 shows the average changes in CAP thresholds for the three experimental groups after the 2 week recovery period. These changes were measured by comparing CAP thresholds after the recovery period with the pretreatment CAP thresholds in the same animals. It is evident that there was no significant effect of the anesthesia, surgery, and recovery period on CAP thresholds in the sham group (Fig. $3 A, B$ ). In contrast, both lesion groups show marked elevations of threshold (Fig. $3 C-F$ ). In the acoustic trauma groups these differences were statistically significant for all frequencies from 10 to $24 \mathrm{kHz}$, with the exception of $16 \mathrm{kHz}$ (Fig. $3 E, F$ ). In the mechanical trauma group, the average pattern of threshold elevation appeared roughly similar to that in the acoustic trauma group, but larger interanimal variation meant that these changes were only significant relative to the sham group at 12 and $14 \mathrm{kHz}$ (Fig. 3C,D).

\section{Spontaneous activity}

SFR was measured in a total of 1118 neurons (263 from the shams, 486 from the mechanical lesions, and 369 from the acoustic trauma groups). Figure 4 shows the data for these three groups pooled regardless of whether or not CF and response category were obtained. There was a significant increase in the mean spontaneous firing rate in both the mechanical lesion and acoustic trauma groups relative to the sham group ( $p<0.001$ for both comparisons).

Figure 5 shows the SFR data for all those neurons whose CF was determined. To assess whether or not SFR increases were related to the regions of peripheral hearing loss, the data were 

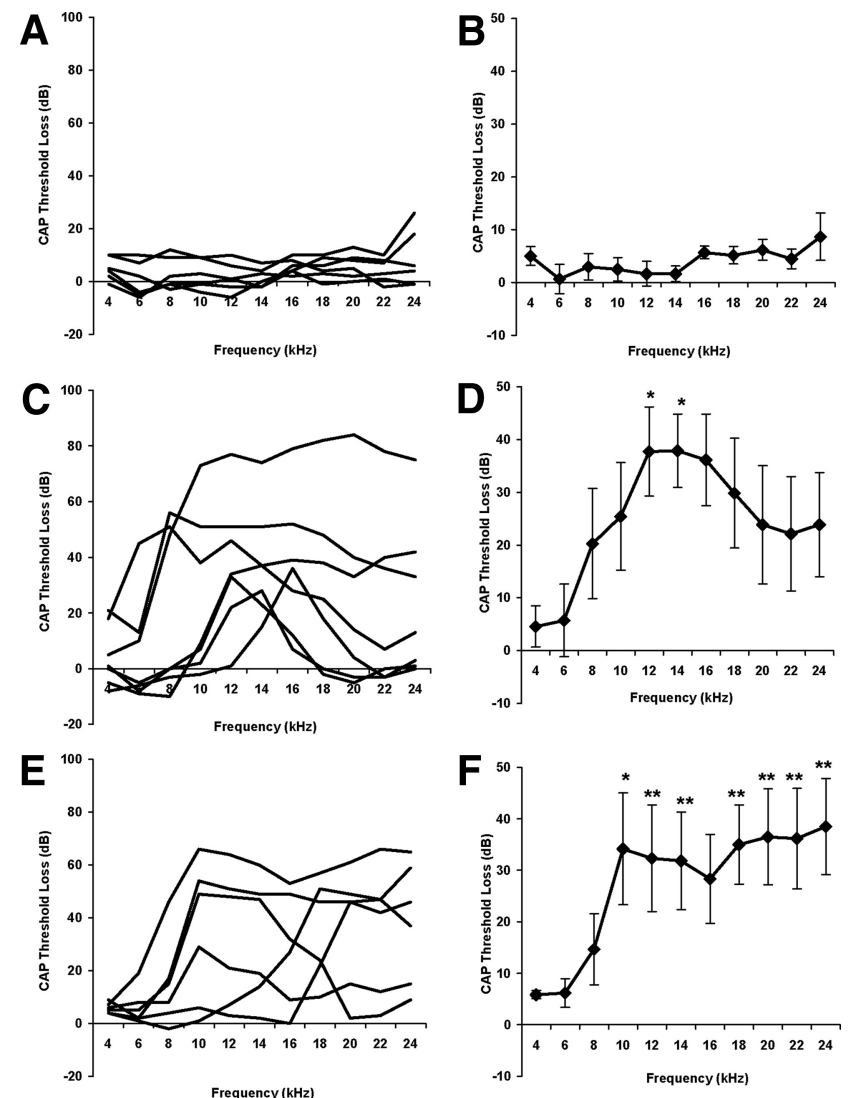

Figure 3. Changes in cochlear sensitivity 2 weeks after initial treatment, measured as change in compound action potential (CAP), thresholds from 4-24 kHz. Left-hand column, Results from individual animals; right-hand column, average data \pm SEM. $\boldsymbol{A}, \boldsymbol{B}$, Sham animals; $\boldsymbol{C}, \boldsymbol{D}$, mechanical lesion; $\boldsymbol{E}, \boldsymbol{F}$, acoustic trauma. ${ }^{*} p<0.05$; ${ }^{* *} p<0.01$.

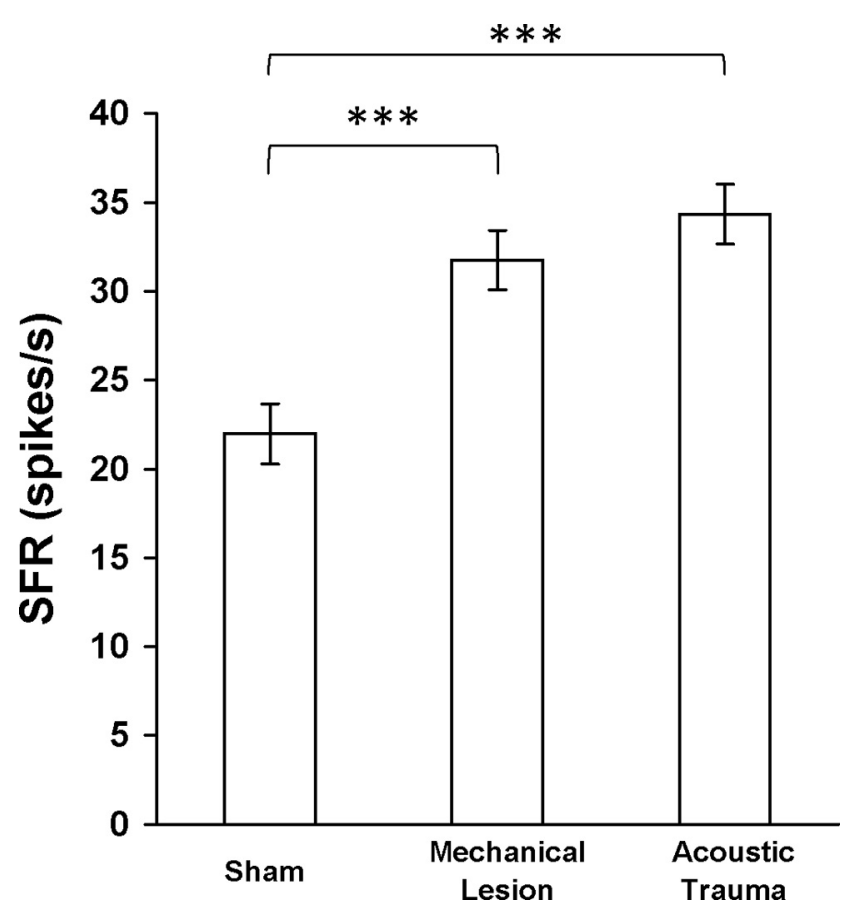

Figure 4. Average spontaneous firing rate (SFR) \pm SEM in the three groups (sham, $n=263$; mechanical lesion, $n=486$; acoustic trauma, $n=369)$. ${ }^{* * *} p<0.001$.

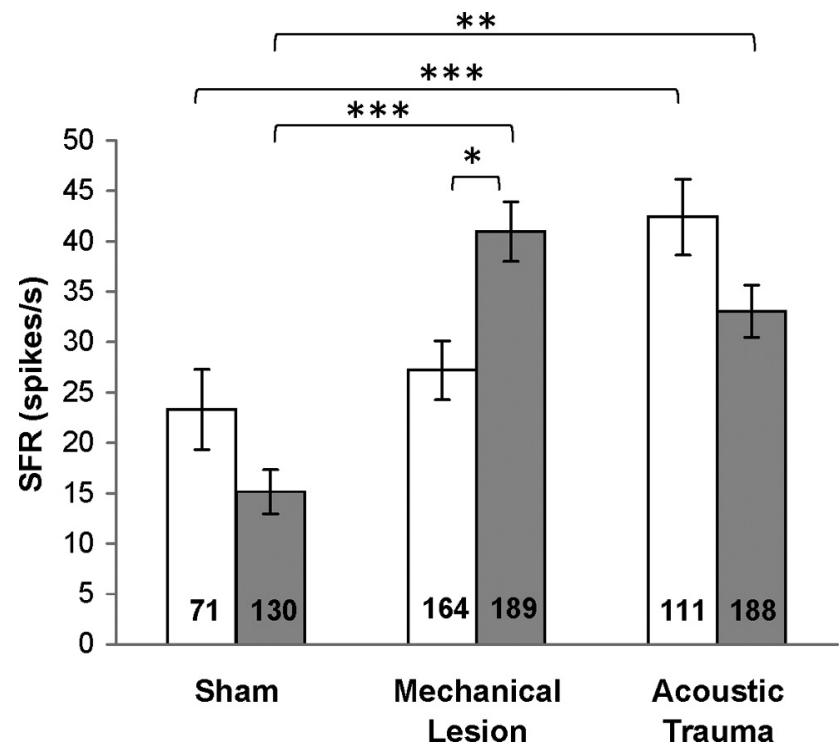

Figure 5. Average spontaneous firing rate (SFR) \pm SEM in the three groups, divided according to CF region. White bars, $\mathrm{CF} \leq 8 \mathrm{kHz}$; gray bars, $\mathrm{CF} \geq 10 \mathrm{kHz}$. ${ }^{*} p<0.05$; ${ }^{* *} p<0.01$; ${ }^{* * *} p<0.001$. Numbers of neurons in each group are shown inside histogram bars. SFR was significantly elevated relative to shams in both CF regions in acoustic trauma, but only in the CF $\geq 10 \mathrm{kHz}$ region in mechanical lesions.

grouped according to two broad $\mathrm{CF}$ ranges $(\leq 8 \mathrm{kHz}$ and $\geq 10$ $\mathrm{kHz})$ so as to correspond roughly to the frequency ranges where the CAP thresholds were approximately normal or substantially elevated. For the purposes of this analysis, neurons with CFs in the range $8-10 \mathrm{kHz}$ were excluded because the interanimal variation in the pattern of hearing loss meant that these frequencies were not consistently either affected or unaffected by the inner ear trauma. A total of 853 neurons were able to be included in this analysis, of which 201 were in the sham group, 353 in the mechanical lesion group, and 299 in the acoustic trauma group.

In the mechanical lesion group, SFR was significantly elevated relative to the sham group only in the CF region corresponding to frequencies at which CAP threshold was consistently elevated. Interestingly, in the acoustic trauma group, SFR was elevated in comparison with sham controls in both CF regions. Hence, SFR increases seem to be more widely distributed across CF regions after acoustic trauma compared to after mechanical lesions.

Assigning a CF can be problematic when recording from neurons in areas of elevated threshold because of the broadening of response areas and also because of the potential for CF shifts (Salvi et al., 1978; Cody and Johnstone, 1980; Liberman, 1984). We therefore used another method of assigning a "nominal" CF based on depth within the VCN. A similar method was used previously in studies of the inferior colliculus (Dong et al., 2009, 2010; Mulders and Robertson, 2009). The depth at which each neuron was encountered was converted to a percentage of distance along each electrode track using the point of first VCN activity as the starting point and the end of the track as the depth when cluster responses abruptly diminished. In each animal an equation was calculated relating the depth along the track to CF. When the correlation coefficient was $>0.5$, nominal CF was calculated. The result was essentially the same as that found previously. Hyperactivity (elevated SFR) was present for neurons in the nominal CF range related to the frequencies of the hearing loss $(\geq 10 \mathrm{kHz})$ in the mechanical lesion and acoustic trauma groups and also in the $0-8 \mathrm{kHz}$ range for the acoustic trauma group. The consistency between the analysis based on grouping 
Table 1. Distribution (\%) of the major response categories in the three animal groups and the number $(n)$ of neurons in each category

\begin{tabular}{llllll}
\hline & Primary-like & Chopper & Onset & Unclassified & Total \\
\hline Sham & & & & & \\
$\quad$ Neurons $(n)$ & 64 & 43 & 30 & 6 & 143 \\
$\quad$ Distribution (\%) & 44.75 & 30.06 & 20.99 & 4.20 & 100 \\
$\quad$ Mechanical lesions & & & & & \\
$\quad$ Neurons ( $n$ ) & 121 & 61 & 59 & 17 & 258 \\
$\quad$ Distribution (\%) & 46.90 & 23.64 & 22.86 & 6.60 & 100 \\
Acoustic trauma & & & & & \\
$\quad$ Neurons (n) & 91 & 36 & 55 & 7 & 189 \\
$\quad$ Distribution (\%) & 48.15 & 19.05 & 29.10 & 3.70 & 100 \\
\hline
\end{tabular}
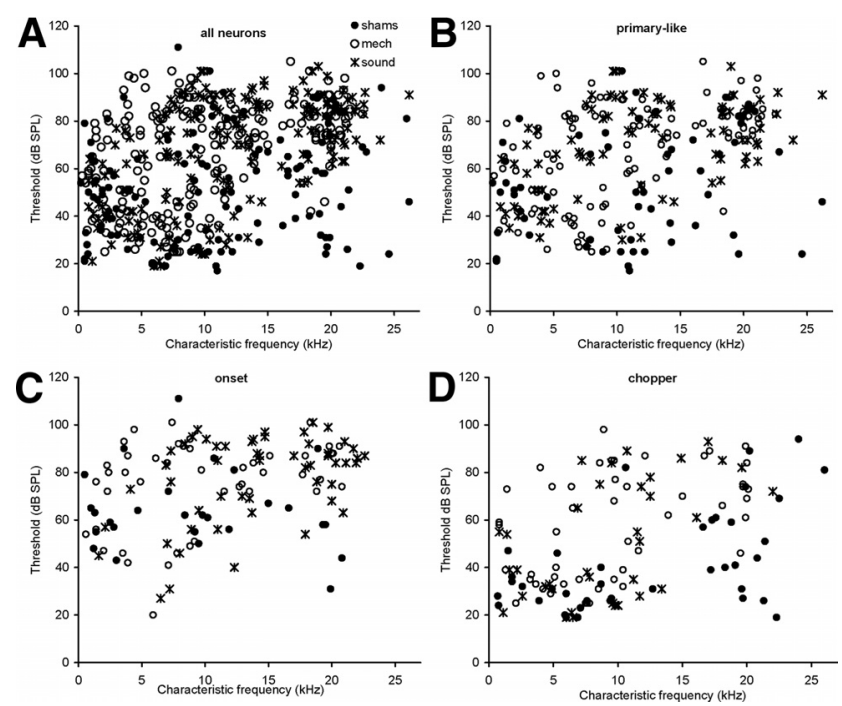

Figure 6. Scatter plots showing characteristic frequency against threshold of all neurons (shams, sham group; mech, mechanical lesion group; sound, acoustic trauma group) $(\boldsymbol{A})$, primary-like neurons $(\boldsymbol{B})$, onset neurons $(\boldsymbol{C})$, and chopper neurons $(\boldsymbol{D})$. Neurons recorded from the different experimental groups are indicated with different symbols.

neurons according to nominal CF and estimated CF indicates that the presence of hyperactivity outside the zone of hearing loss after acoustic trauma was unlikely to be attributed to incorrect estimation of CF.

Neuron response categories

A total of 590 neurons were able to be reliably classified according to response type (143 in sham, 258 in mechanical lesion, and 189 in acoustic trauma groups). The percentage of neurons of each cell type was compared between the different conditions. The data are summarized in Table 1. A $\chi^{2}$ analysis showed no significant difference in the percentage of cell types between the three groups. Hence there is no evidence that the cochlear trauma caused alterations in the distribution of response types of neurons in the VCN. Figures $6 \mathrm{~A}$ and $7 \mathrm{~A}$ show scatter plots of the characteristic frequency of all these 590 neurons against their threshold and spontaneous activity, respectively. In Figures $6 B-D$ and $7 B-D$, scatter plots are shown after neurons were allocated to a response category.

When the SFR data were grouped according to response type, the increase in SFR seen in the two treatment groups compared to shams was more obvious in some response categories than in others. Figure $8 \mathrm{~A}$ shows that a statistically significant rise in SFR was found in the PL and $\mathrm{O}$ categories when acoustic trauma and sham groups were compared. However, no statistically signifi-

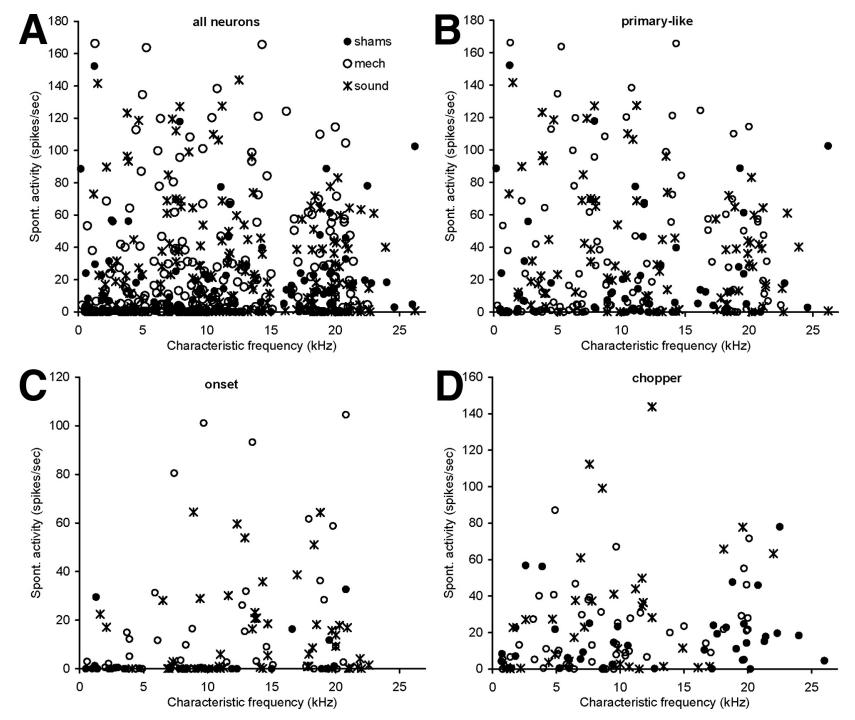

Figure 7. Scatter plots showing characteristic frequency against spontaneous activity of all neurons (shams, sham group; mech, mechanical lesion group; sound, acoustic trauma group) (A), primary-like neurons $(\boldsymbol{B})$, onset neurons $(\boldsymbol{C})$ and chopper neurons $(\boldsymbol{D})$. Neurons recorded from the different experimental groups are indicated with different symbols.

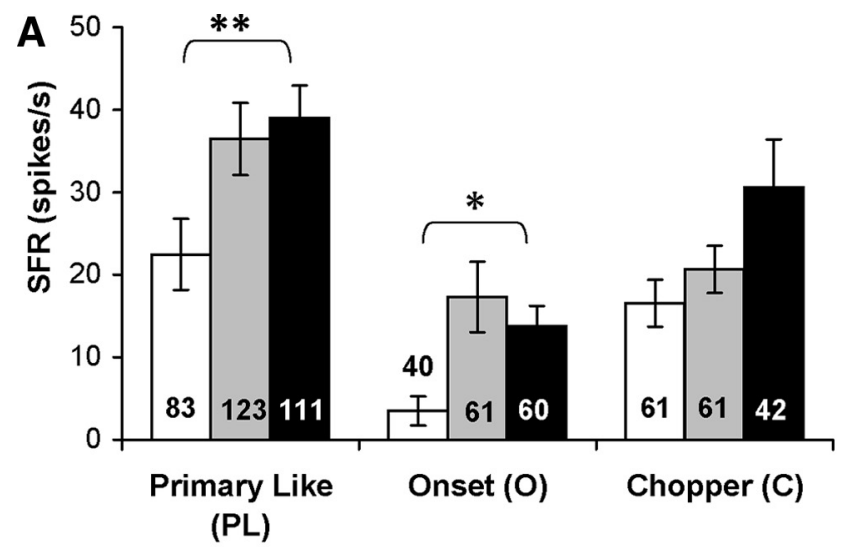

B

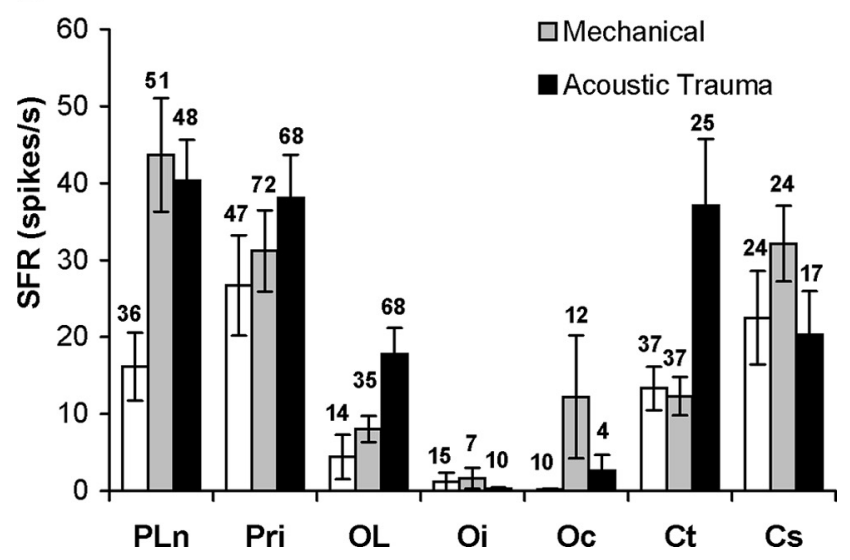

Figure 8. Average spontaneous firing rate (SFR) in the different experimental groups grouped according to response category. $\boldsymbol{A}$, Three main categories. Note significant hyperactivity relative to shams in PL and 0 categories in the acoustic trauma group. $\boldsymbol{B}$, More fine grain grouping into subcategories. Numbers of neurons in each group are shown inside histogram bars in $\boldsymbol{A}$ and above bars in $\boldsymbol{B} .{ }^{*} p<0.05 ;{ }^{* *} p<0.01$. Pln, Primary-like with notch; Pri, primary-like; 0 c, onset chopper; $\mathrm{Ct}$, transient chopper; $\mathrm{C}$, sustained chopper. 
cant increase was found for the $\mathrm{C}$ category. A similar pattern was present for the mechanical lesion group compared to shams, but the changes did not reach statistical significance. The data were further broken down into subcategories (Fig. $8 \mathrm{~B}$ ). Hyperactivity appeared to be most prominent in the PLn, OL, and Oc subcategories and in the Ct group. However, none of these differences was significant, presumably because of the relatively small sample sizes in the subgroups compared to the pooled data in the overarching PL, O, and C categories. A similar problem was encountered when the SFR in the different response categories was examined according to CF range. Although differences were seen that were similar to those seen in the pooled data shown in Figure 5 , none of these differences reached statistical significance (data not shown).

\section{Discussion}

The present data show a significant increase in average spontaneous firing rates in the VCN after cochlear trauma that leads to lasting changes in peripheral sensitivity. Our demonstration of hyperactivity in VCN at the single cell electrophysiological level is consistent with more indirect evidence using magnetic resonance imaging and assays of excitatory neurotransmitter enzymes (Zheng et al., 2006; Brozoski et al., 2007). Hence, the DCN is not the only subdivision of the $\mathrm{CN}$ that shows neuronal hyperactivity after treatments that are associated with the development of tinnitus in both human and animal subjects. The present experiments do not allow us to rule out the possibility that the hyperactivity in VCN is in fact a consequence of hyperactivity in the DCN mediated by interconnections between these two subdivisions. It must be considered equally possible that hyperactivity reported in DCN is driven by hyperactivity in VCN. However, available evidence suggests that the main interconnections between DCN and VCN are inhibitory in nature (see for example, Doucet and Ryugo, 1997; Wickesberg and Oertel, 1990) and are unlikely to mediate hyperactivity in either structure as a result of hyperactivity in the other.

Regardless, the results show that the VCN must now be considered when speculating about the role of lower brainstem structure centers in the development and maintenance of hyperactivity in higher auditory centers such as the inferior colliculus and auditory cortex. For example, although hyperactivity has been amply demonstrated in the DCN (Kaltenbach and Afman, 2000; Kaltenbach et al., 2000, 2004; Brozoski et al., 2002; Kaltenbach, 2007), it has been shown in an animal model that tinnitus persists after DCN ablation (Brozoski and Bauer, 2005). This result has been interpreted as evidence that the neural tinnitus generators eventually become established in higher centers independent of abnormal firing in the lower brainstem. However, if the VCN as well as the DCN exhibits hyperactivity after cochlear trauma, then the cochlear nucleus still cannot be ruled out as a possible source of persistent hyperactive drive to higher centers. The inferior colliculus in particular, which has been consistently shown to exhibit hyperactivity (Ma et al., 2006; Dong et al., 2009; Mulders and Robertson, 2009; Dong et al., 2010; Mulders et al., 2010), receives substantial input from VCN directly via $\mathrm{T}$ stellate neurons and indirectly via T stellate, bushy cell, and octopus cell projections to the superior olivary complex and lateral lemniscal nuclei (Beyerl, 1978; Adams, 1979; BrunsoBechtold et al., 1981; Coleman and Clerici, 1987; Cant and Benson, 2003; Smith et al., 2005).

The different response categories of VCN neurons have different patterns of projection to higher stages of the auditory pathway and different roles in auditory processing. Hence, it is of interest that the present data show that hyperactivity in the VCN is not uniform across all cell types, being most prominent in $\mathrm{PL}$ and $\mathrm{O}$ response categories. This is reminiscent of the situation in DCN in which it has been shown that hyperactivity after cochlear trauma is confined to the fusiform cells that form the main output neurons of the DCN (Brozoski et al., 2002). These data together reinforce that view that hyperactivity is a cell-specific change in synaptic and/or membrane properties of the central neurons resulting from the altered input caused by the peripheral trauma. Although we attempted to determine whether particular subcategories of response type were more hyperactive than others, the sample size proved inadequate for such a fine grain analysis.

A further intriguing aspect of the present data is that the distribution of hyperactivity across CF regions in the VCN appeared to differ from that seen in other tonotopically organized structures. In the inferior colliculus after both acoustic trauma and mechanical cochlear lesions, hyperactivity is more or less confined to CF regions that are contained within the frequency regions of the persistent peripheral hearing loss (Dong et al., 2009, 2010; Mulders and Robertson, 2009). In the DCN as well, it has been reported that elevated background neural ensemble activity is seen in parts of the DCN related to the frequency of a traumatizing sound (Kaltenbach and Afman, 2000; Kaltenbach et al., 2000). In the present study of VCN, however, this was only true in the mechanical lesion group. The acoustic trauma group, in contrast, showed a more widespread increase in SFR that was evident in CF regions much lower than those at which cochlear thresholds were markedly elevated. The reason for more widespread hyperactivity in the VCN after acoustic trauma is unclear, but several factors may be involved. Direct mechanical lesions to hair cells and nerve fibers presumably produce immediate reduction in afferent activity. Acoustic trauma on the other hand is accompanied, for at least a short period during the early stages of the exposure, by a large increase in primary afferent firing. In addition, the acute change in threshold at the end of an acoustic trauma is more widespread that that produced by mechanical lesions, although much of this returns to normal during the recovery period (Dong et al., 2009, 2010; Mulders and Robertson, 2009). The hyperactivity seen in lower CF regions of the VCN after a $10 \mathrm{kHz}$ acoustic trauma may reflect these differences, but this does not explain why similar effects are not seen in DCN or why the more widespread hyperactivity in VCN as a result of acoustic trauma does not appear to be reflected in a similar distribution in higher centers such as the inferior colliculus (Dong et al., 2009, 2010; Mulders and Robertson, 2009). It may be that particular VCN projections to higher centers transmit hyperactivity more faithfully than others. For example, some categories of onset neurons are known to be inhibitory (Smith and Rhode, 1989; Ferragamo et al., 1998; Doucet et al., 1999; Babalian et al., 2002; Arnott et al., 2004), and their hyperactivity might not give rise to hyperactivity in corresponding target regions in the inferior colliculus. Furthermore, some PL neurons project to inhibitory neurons in superior olivary complex and ventral nucleus of the lateral lemniscus (Cant and Benson, 2003). The number of neurons obtained in this study was not sufficient to demonstrate clear differences in the CF distribution of hyperactivity for different response categories that correspond to different ascending projection patterns from the VCN.

\section{References}

Adams JC (1979) Ascending projections to the inferior colliculus. J Comp Neurol 183:519-538. 
Arnott RH, Wallace MN, Shackleton TM, Palmer AR (2004) Onset neurones in the anteroventral cochlear nucleus project to the dorsal cochlear nucleus. J Assoc Res Otolaryngol 5:153-170.

Babalian AL, Jacomme AV, Doucet JR, Ryugo DK, Rouiller EM (2002) Commissural glycinergic inhibition of bushy and stellate cells in the anteroventral cochlear nucleus. Neuroreport 13:555-558.

Bauer CA, Turner JG, Caspary DM, Myers KS, Brozoski TJ (2008) Tinnitus and inferior colliculus activity in chinchillas related to three distinct patterns of cochlear trauma. J Neurosci Res 86:2564-2578.

Beyerl BD (1978) Afferent projections to the central nucleus of the inferior colliculus in the rat. Brain Res 145:209-223.

Brozoski TJ, Bauer CA (2005) The effect of dorsal cochlear nucleus ablation on tinnitus in rats. Hear Res 206:227-236.

Brozoski TJ, Bauer CA, Caspary DM (2002) Elevated fusiform cell activity in the dorsal cochlear nucleus of chinchillas with psychophysical evidence of tinnitus. J Neurosci 22:2383-2390.

Brozoski TJ, Ciobanu L, Bauer CA (2007) Central neural activity in rats with tinnitus evaluated with manganese-enhanced magnetic resonance imaging (MEMRI). Hear Res 228:168-179.

Brunso-Bechtold JK, Thompson GC, Masterton RB (1981) HRP study of the organization of auditory afferents ascending to central nucleus of inferior colliculus in cat. J Comp Neurol 197:705-722.

Cant NB, Benson CG (2003) Parallel auditory pathways: projection patterns of the different neuronal populations in the dorsal and ventral cochlear nuclei. Brain Res Bull 60:457-474.

Cody AR, Johnstone BM (1980) Single auditory neuron response during acute acoustic trauma. Hear Res 3:3-16.

Coleman JR, Clerici WJ (1987) Sources of projections to subdivisions of the inferior colliculus in the rat. J Comp Neurol 262:215-226.

Dong S, Mulders WH, Rodger J, Robertson D (2009) Changes in neuronal activity and gene expression in guinea-pig auditory brainstem after unilateral partial hearing loss. Neuroscience 159:1164-1174.

Dong S, Mulders WH, Rodger J, Woo S, Robertson D (2010) Acoustic trauma evokes hyperactivity and changes in gene expression in guinea-pig auditory brainstem. Eur J Neurosci 31:1616-1628.

Doucet JR, Ryugo DK (1997) Projections from the ventral cochlear nucleus to the dorsal cochlear nucleus in rats. J Comp Neurol 385:245-264.

Doucet JR, Ross AT, Gillespie MB, Ryugo DK (1999) Glycine immunoreactivity of multipolar neurons in the ventral cochlear nucleus which project to the dorsal cochlear nucleus. J Comp Neurol 408:515-531.

Ferragamo MJ, Golding NL, Oertel D (1998) Synaptic inputs to stellate cells in the ventral cochlear nucleus. J Neurophysiol 79:51-63.

Imig TJ, Durham D (2005) Effect of unilateral noise exposure on the tonotopic distribution of spontaneous activity in the cochlear nucleus and inferior colliculus in the cortically intact and decorticate rat. J Comp Neurol 490:391-413.

Johnstone JR, Alder VA, Johnstone BM, Robertson D, Yates GK (1979) Cochlear action potential threshold and single unit thresholds. J Acoust Soc Am 65:254-257.

Kaltenbach JA (2007) The dorsal cochlear nucleus as a contributor to tinnitus: mechanisms underlying the induction of hyperactivity. Prog Brain Res 166:89-106.

Kaltenbach JA, Afman CE (2000) Hyperactivity in the dorsal cochlear nucleus after intense sound exposure and its resemblance to tone-evoked activity: a physiological model for tinnitus. Hear Res 140:165-172.

Kaltenbach JA, Zhang J, Afman CE (2000) Plasticity of spontaneous neural activity in the dorsal cochlear nucleus after intense sound exposure. Hear Res 147:282-292.
Kaltenbach JA, Zacharek MA, Zhang J, Frederick S (2004) Activity in the dorsal cochlear nucleus of hamsters previously tested for tinnitus following intense tone exposure. Neurosci Lett 355:121-125.

Komiya H, Eggermont JJ (2000) Spontaneous firing activity of cortical neurons in adult cats with reorganized tonotopic map following pure-tone trauma. Acta Otolaryngol 120:750-756.

Liberman MC (1984) Single-neuron labeling and chronic cochlear pathology. I. Threshold shift and characteristic-frequency shift. Hear Res 16:33-41.

Ma WL, Hidaka H, May BJ (2006) Spontaneous activity in the inferior colliculus of $\mathrm{CBA} / \mathrm{J}$ mice after manipulations that induce tinnitus. Hear Res 212:9-21.

Merrill EG, Ainsworth A (1972) Glass-coated platinum-plated tungsten microelectrodes. Med Biol Eng 10:662-672.

Mulders WH, Robertson D (2009) Hyperactivity in the auditory midbrain after acoustic trauma: dependence on cochlear activity. Neuroscience 164:733-746.

Mulders WH, Harvey AR, Robertson D (2007) Electrically evoked responses in onset chopper neurons in guinea pig cochlear nucleus. J Neurophysiol 97:3288-3297.

Mulders WH, Seluakumaran K, Robertson D (2010) Efferent pathways modulate hyperactivity in inferior colliculus. J Neurosci 30:9578-9587.

Rhode WS, Smith PH (1986) Encoding timing and intensity in the ventral cochlear nucleus of the cat. J Neurophysiol 56:261-286.

Rhode WS, Smith PH, Oertel D (1983) Physiological response properties of cells labeled intracellularly with horseradish peroxidase in cat dorsal cochlear nucleus. J Comp Neurol 213:426-447.

Robertson D, Cody AR, Bredberg G, Johnstone BM (1980) Response properties of spiral ganglion neurons in cochleas damaged by direct mechanical trauma. J Acoust Soc Am 67:1295-1303.

Salvi RJ, Hamernik RP, Henderson D (1978) Discharge patterns in the cochlear nucleus of the chinchilla following noise induced asymptotic threshold shift. Exp Brain Res 32:301-320.

Seki S, Eggermont JJ (2003) Changes in spontaneous firing rate and neural synchrony in cat primary auditory cortex after localized tone-induced hearing loss. Hear Res 180:28-38.

Smith PH, Rhode WS (1989) Structural and functional properties distinguish two types of multipolar cells in the ventral cochlear nucleus. J Comp Neurol 282:595-616.

Smith PH, Massie A, Joris PX (2005) Acoustic stria: anatomy of physiologically characterized cells and their axonal projection patterns. J Comp Neurol 482:349-371.

Wickesberg RE, Oertel D (1990) Delayed, frequency-specific inhibition in the cochlear nuclei of mice: a mechanism for monaural echo suppression. J Neurosci 10:1762-1768.

Winter IM, Palmer AR (1990) Responses of single units in the anteroventral cochlear nucleus of the guinea pig. Hear Res 44:161-178.

Winter IM, Palmer AR (1995) Level dependence of cochlear nucleus onset unit responses and facilitation by second tones or broadband noise. J Neurophysiol 73:141-159.

Young ED, Robert JM, Shofner WP (1988) Regularity and latency of units in ventral cochlear nucleus: implications for unit classification and generation of response properties. J Neurophysiol 60:1-29.

Zheng Y, Seung Lee H, Smith PF, Darlington CL (2006) Neuronal nitric oxide synthase expression in the cochlear nucleus in a salicylate model of tinnitus. Brain Res 1123:201-206. 